\title{
Concept Based Approach for Adaptive Personalized Course Learning System
}

\author{
Mehmet Ali SALAHLI ${ }^{1}$, Muzaffer ÖZDEMIR ${ }^{1} \&$ Cumali YAŞAR ${ }^{1}$ \\ ${ }^{1}$ Department of Computer and Instructional Technologies Education, Çanakkale Onsekiz Mart University, \\ Turkey \\ Correspondence: Mehmet Ali SALAHLI, Department of Computer and Instructional Technologies Education, \\ Çanakkale Onsekiz Mart University, Turkey. Tel: 90-537-774-8420. E-mail: msalahli@comu.edu.tr
}

Received: February 26, 2013 Accepted: March 18, 2013 Online Published: April 27, 2013

doi:10.5539/ies.v6n5p92

URL: http://dx.doi.org/10.5539/ies.v6n5p92

This research is financed by the Research Found of Canakkale Onsekiz Mart University, Turkey under the Project No 18/2011.

\begin{abstract}
One of the most important factors for improving the personalization aspects of learning systems is to enable adaptive properties to them. The aim of the adaptive personalized learning system is to offer the most appropriate learning path and learning materials to learners by taking into account their profiles. In this paper, a new approach to adaptive personalized e-learning systems is proposed. This approach provides navigation on course materials, as well as navigations on course topics and domain concepts. In this approach, in addition to the difficulty of learning materials and course topics, students' levels of knowledge and students' understanding degrees on the course topics have also been taken into account. The Item Response Theory and Law of Total Probability have been used for estimating understanding degrees. The performance evaluation of the proposed approach has been tested by using the exam and project results, as well as grade point averages of the students from the Computer and Instructional Technology Department. The test results show the accuracy of the proposed method. It is believed that this study can improve the effectiveness of the adaptive e-learning system.
\end{abstract}

Keywords: personalized learning, adaptive learning, e-learning, understanding degree

\section{Introduction}

One of the most important factors for improving the personalization aspects of learning systems is to enable adaptive properties to them. The aim of the adaptive personalized learning system is to offer the most appropriate learning path and learning materials to learners by taking into account theirs profiles. These systems enhance the usability of learning materials, and thus make the e-Learning system more effective. As a consequence, the learners' acquisition of knowledge will be improved; this will lead to a more effective learning process (Esichaikul et al., 2011). In the adaptive content aggregation method proposed in Mödritscher et al. (2004), different types of content that depend on the learning and teaching style of a learner are offered; whereas adaptive presentation can be enriched with additional explanations, adaptive navigation can be adapted through global or local guidance and orientation.

The paper Muñoz and Oliveira (2004) describes the main steps taken in developing an Adaptive Web Training Environment, which consists of the LOM standard, Domain and Student Knowledge Models, and Web ontologies. The Web-based Intelligent Tutoring System, which allows students for a dynamic generation of suitable courseware and provides adaptive feedback, is presented in Kosba and Boyle, (2003)

Methods and Models based on the Probabilistic theory are widely used in the development of tutoring systems. Detailed information on the related topic can be found in the Woolf (2009). A Personalized e-learning system using the Item Response Theory is proposed in Shih and Tseng (2009). To recommend learners appropriate course materials that are based on their individual requirements, the authors use the item characteristic function with difficulty parameters. The idea, presented in this paper is improved in Chen and Duh (2008). The Fuzzy item response theory, which is considered to be capable of helping to recommend courseware with suitable difficulty levels for learners according to learner's fuzzy feedback responses, is proposed by the authors of this 
study. Authors of the study Baylari and Montazer (2009) propose a personalized multi-agent e-learning system which can be used to estimate learner's ability by means of the item response theory, and then to present a personalized and adaptive post-test based on that ability. Also the system can assist the discovery of learner's learning problems via learner responses on review tests through using an artificial neural network (ANN) approach and then appropriate learning materials can be recommended to the student.

The proposed framework in the Dağ and Erkan (2010) is aimed to form a personalized learning environment. The designed learning system according to this framework includes the domain model, user model, and adaptation model. The components of the system have been realized by an ontology-based knowledge modelling approach. The problems related to the integration of learning standards, Semantic Web, and adaptive technologies to meet the learner requirements, are discussed in Jovanovic at al. (2009).

As a result of a brief review on adaptive personalized systems, we can conclude that, in most systems, adaptability is achieved by providing appropriate course materials to learners in accordance with their profiles. Meanwhile, course materials are not the only factor influencing learning outcomes. One of the reasons that the learner does not understand the courseware may be that he/she does not have enough prerequisite knowledge about the topic to be learned. Unfortunately, one way of adapting this aspect is often neglected.

The approach proposed in this paper takes into account the difficulty of learning materials and course topics, students' knowledge levels as well as their understanding degrees of the course topics; and it also provides with a navigation of the course materials, as well as navigations of the course topics and domain concepts. The approach makes use of the Item Response Theory and The Law of Total Probability for estimate of understanding degrees. The offered approach has been carried out in the Adaptive Learning Management System developed by the authors.

The paper has been structured as follows: The background knowledge on the research subject is given in Section 2. In Section 3, the proposed approach is explained. Some experimental results related to the subject area are analysed in Section 4. Finally, the conclusion is given in Section 5.

\section{The Background Knowledge}

\subsection{Item Response Theory}

The Item response theory (IRT) is a probabilistic model, which gives the probability that a person with a given ability level will answer correctly (Baker, 2001). The simplest form of IRT is the One-parameter Logistic Model, also known as the Rasch Model (Baker), which posits that the probability of a person with ability $\theta$, answering an item with difficulty $b$ correctly depends on both the ability of the person and difficulty of the item. In other words, if a person has a high ability in a particular field, he or she will probably get an easy item correct.

The Rasch model is expressed by the following formula:

$$
P_{i}(\theta)=\frac{e^{D\left(\theta-b_{i}\right)}}{1+e^{\left(\theta-b_{i}\right)}}
$$

where $\mathrm{P}_{\mathrm{i}}(\theta)$ is the probability that a randomly chosen examinee with ability $\theta$, answers the item $i$ correctly;

$e$ is a mathematical constant, approximately equal to 2.71828 , that is the base of the natural logarithm;

$\theta$ is the ability of a person;

$\mathrm{b}_{\mathrm{i}}$ is a difficulty parameter of the item $i$, and

$D$ is a scaling constant usually set equal to 1.702

\subsection{The Law of Total Probability}

In the probability theory, the law of total probability is a fundamental rule, relating marginal probabilities to conditional probabilities. The law of total probability is the proposition that if $\left\{B_{n_{-}} \mathrm{n}=1,2,3, \ldots\right\}$ is a finite partition of a sample space,_and for each event $B_{n}$ - is measurable, then the probability of any event $A$ of the same probability space is:

$$
\mathrm{P}(\mathrm{A})=\sum_{n}\left(P\left(A / B_{n}\right) P\left(B_{n}\right)\right.
$$

where $P\left(A / B_{n}\right)$ is the conditional probability of event $A$ on $B_{n}$;

$P\left(B_{n}\right)$ - The occurrence probability of event $B_{n}$ (Wikipedia , 2012).

\subsection{Ontology}

Gruber (1993) defines ontology as "the specification of a conceptualization". Therefore, ontology is a 
description of concepts and their relationships. It permits the structuring of a knowledge domain. Ontologies are commonly used for describing the metadata of learning resources in order to help with the searching of learning objects in data repositories. Course ontology is used to describe the relations among course subjects. The ontology can be expressed as a graph $G(T, R)$, where $T$ is a set of the nodes which represent the subjects, and $R$ is a set of semantic relationships between the pairs of nodes $t$ and $k(\mathrm{t}, \mathrm{k} \in \mathrm{T})$. Each edge can be assigned a weight. The fragment of the graphical depiction of the subject ontology on the course of Database Management Systems (DBMS) is given in Figure 1.

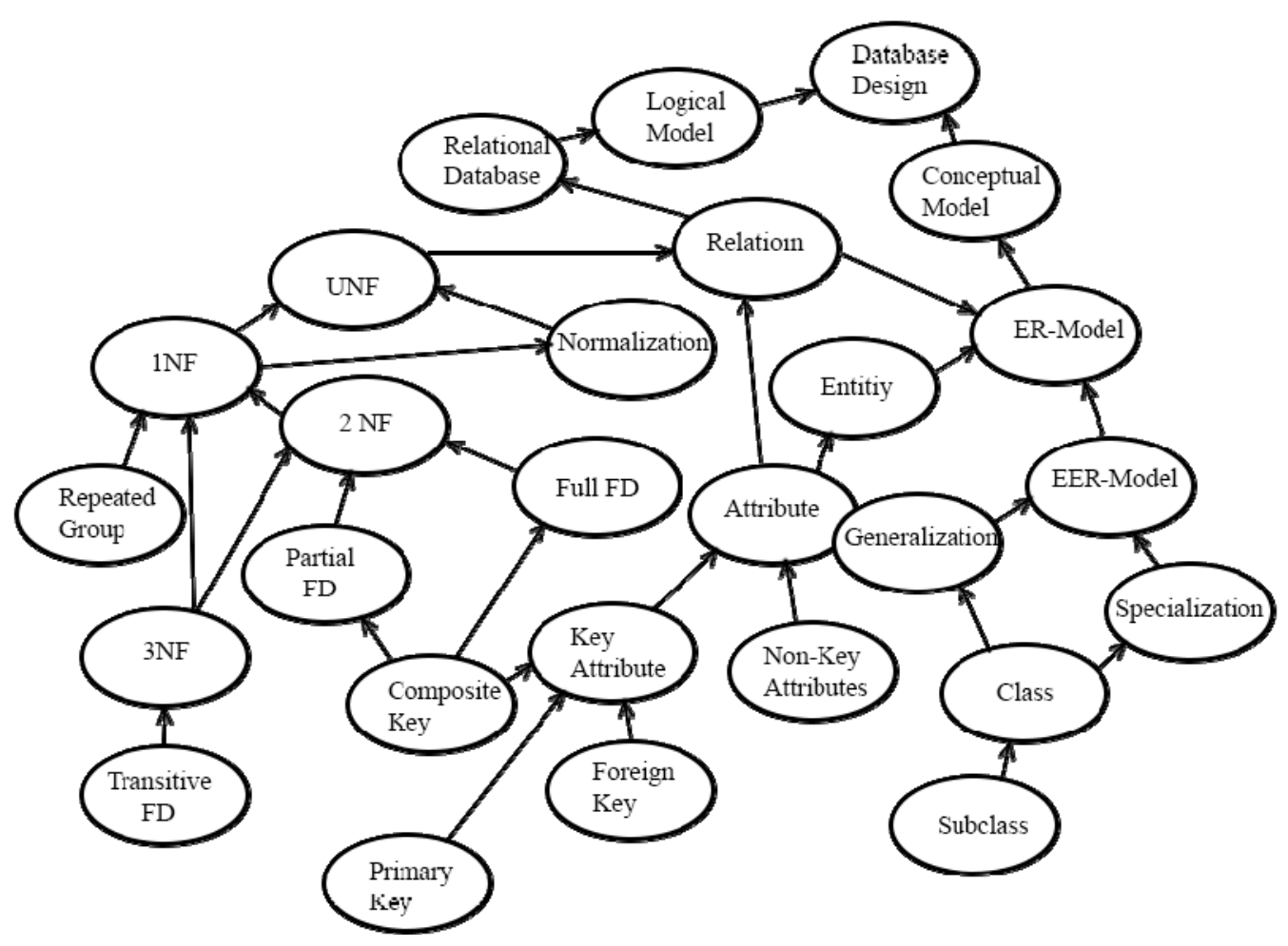

Figure 1. Graphic representation of the concept ontology for the DBMS course

\section{Definitions of some concepts used in the suggested method}

Definition 1. Difficulty level of a learning material: $D M^{i}$ represents the difficulty level of the $i$ th learning material. According to the LOM standard, the difficulty of a learning object is determined according to "how hard it is to work with or through this learning object for the typical intended target audience" (IEEE, 2004). To evaluate the difficulty level of a learning object, the Standard recommends 5 linguistic values: \{very easy, easy, medium, difficult, very difficult\}. Hereafter, we will use the abbreviations of these terms as \{VE, E, M, D, VD\}, respectively.

Definition 2. Difficulty level of course concept: $\mathrm{DC}^{\mathrm{j}}$ represents the difficulty level of the $j$ th course topic. We use this parameter to determine "how difficult it is to understand this course concept". This parameter takes a value from the set of $\{\operatorname{very} \operatorname{easy}(V E)$, easy $(E)$, medium $(M)$, difficult(D), very $\operatorname{difficult}(V D)\}$.

Definition 3. Student's Knowledge Level: $\mathrm{KWL}_{\mathrm{i}}$ shows the knowledge level of the $i$ th student. This parameter represents the knowledge level of a student on the subject area that he/she wants to learn. The results obtained with different knowledge assessment methods, such as the students' grade point average (GPA), exam grades, and preliminary test results can be used to determine the level of knowledge. We will use five levels of knowledge, namely \{very low $(V L)$, low $(L)$, medium $(M)$, high $(H)$, very high $(V H)\}$.

Definition 4. Understanding degree of course topic: This parameter determines how much and to what extent the topic was understood by the target student.

The understanding degree of the student $j$ of topic $k-U_{j}^{k}$ can be expressed as

$$
U_{j}^{k}=f\left(K W L_{j}, D M^{i}, D C^{k}\right)
$$


Where $K W L_{j}$ is the knowledge level of student $\mathrm{j}$;

$D M^{i}$ represents the difficulty of learning material i as recommended for the study of topic $k$;

$D C^{k}$ is the difficulty of the topic $\mathrm{k}$. In the proposed method this parameter is associated with the key concept of the topic. . Key concepts are determined by domain experts.

The formula (3) means that understanding degree of course topic depends on the following three factors: student's knowledge, difficulty of the concepts referred in the topic, and difficulty of the appropriate learning material.

The process of estimating of understanding degrees is described in Figure 2.

We use the following five linguistic values to estimate understanding degree: \{very low $(V L)$, low $(L)$, medium $(M)$, high $(H)$, very high $(V H)\}$.

After the necessary primary knowledge is given, we can describe the approach for Adaptive Personalized Course Learning System (APCL).

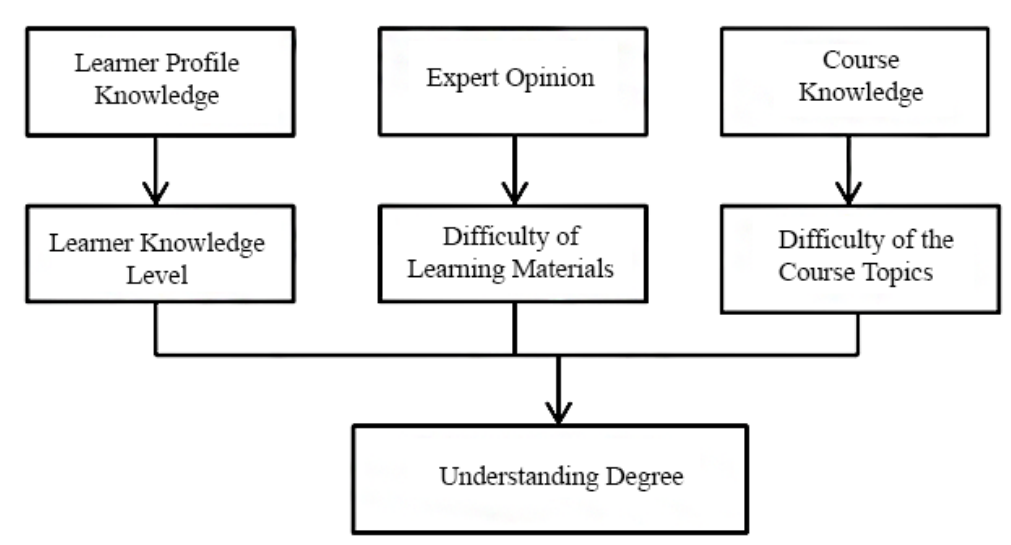

Figure 2. Estimating of understanding degree

\section{The Approach for Adaptive Personalized Course Learning System}

This approach allows for choosing an effective way of learning with regard to parameters such as students' knowledge level, and understanding degree, as well as the difficulty of course topics, and course learning materials. APCL is based on the use of ontological knowledge about course contents, and also on the application of the probabilistic methods, such as the Item Response Theory, and Law of Total Probability.

A brief description of the proposed APCL is given below.

The initial values of understanding degrees are determined for each knowledge level of each course topic. For this purpose, the IRT model is used. The value of understanding degree on the topic is recalculated by means of the Law of Total Probability, as based on the appropriate values of the previous topics,

The implementation process of the approach consists of the following steps.

Step 1. A Knowledge Base is created for the APCL. The implementation of the approach requires some information about learners' profiles, courses to be learned, and learning materials on each course topic. The following steps should be taken to create the knowledge base that consists of this information:

- the course outline, which reflects the hierarchical structure of the course topics to be learned, is determined;

- for each topic, the key concepts are determined, and as a consequence the list of all concepts for the target course are formed;

- a concept ontology, which covers all these concepts and reflects the relationship between them, is created;

- for each course, a list of learning materials is created. Difficulty levels of these materials are determined and the learning materials are ordered by this parameter. 
Step 2. By using the Item Response Theory (IRT) method, the understanding degrees of the course topics for each knowledge level are estimated. We make the assumption that the knowledge is used to measure a student's ability. Due to the fact that knowledge levels and difficulty levels are expressed in linguistic values, it is also needed to convert these values to the appropriate numeric values. For this purpose, the simple conversion tables (Table 1 and Table 2) are used.

Table 1. Linguistic and numeric values for Knowledge Levels

\begin{tabular}{cccccc}
\hline Linguistic & VL & L & M & H & VH \\
Numeric & -2 & -1 & 0 & 1 & 2 \\
\hline
\end{tabular}

Table 2. Linguistic and numeric values for Difficulty Levels

\begin{tabular}{cccccc}
\hline Linguistic & VE & E & M & D & VD \\
Numeric & -2 & -1 & 0 & 1 & 2 \\
\hline
\end{tabular}

Step 3. A student logins the System and enters the title of the topic that he wants to learn. The values of student's knowledge level and the difficulty of the topic are retrieved from the Knowledge Base according to the student's identity information, and to the topic. On the basis of these data, the value of the appropriate understanding degree is determined.

Step 4. If the understanding degree of the student is low, the System offers to the student the learning material with lower difficulty, if not, the learning process is continued on the original list of the learning materials.

Step 5. The student begins to learn the course topic from the recommended learning material.

Step 6. The knowledge of the student on the topic is tested. On the basis of the test results, the student's knowledge level is re-evaluated.

Step 7. If the student wants to learn the next topic, the system re-calculates the understanding degree on the topic, taking into account the new value of the knowledge level. This step is carried out by the LTP module

Step 8. If the understanding degree is low, then the system recommends the student to improve his/her knowledge on the predecessor concepts. The list of the concepts is determined through the concept ontology. The student may decide to go to the study of the next topic or to improve their prerequisite knowledge.

Assume that the student learns a topic, for which the keyword is " $3 N F$ ". The understanding degree of the student with knowledge level "medium" is equal to 0.3. As the understanding degree is low, the system encourages the student to improve their knowledge by learning the predecessor concepts $1 N F$, 2NF, Functional Dependency, Transitive Dependency, and Key Attributes.

Step 9. The student starts studying the new topic.

Step 10. The aim of this step is to determine whether the student has understood the reading material or not. This aim is achieved through an interactive query. The System helps the learner to answer questions such as "what should I read to learn the topic", "what should I do if the proposed material is difficult to understand or materials offered to me are very simple".

When the learner completes the reading of the offered material, the System asks the student whether he/she has understood the content of the material or not. Considering that the answer is positive, the system offers him/her some test materials to measure his/her knowledge level on the related topic. By taking into account the new value of the knowledge level, the understanding degree is re-calculated through the Law of Total Probability. Taking into consideration the new value of the understanding degree, the System gives advice to the students on proceeding with the next topic or not. If the value of understanding degree is low, the system makes suggestions about topics as well as to what extent the student should increase his/her knowledge level. This action can be interpreted as that while increasing knowledge on the previous related topics, the probability of understanding a topic increases.

If the answer of the student is negative, the system tries to identify why student cannot understand the learning material. For this purpose, the system offers the student the following four options: 
a) The material is very theoretical; it could have been clearer with practical examples;

b) I could not understand the following terms (the terms are marked or entered by the learner);

c) I could not understand a particular part of the material (the related part of the learning material is marked);

\section{d) I didn't understand anything}

Case (a): In this case the system offers a new learning material from the ordered list of the learning materials.

Case (b): The system selects the learning objects, from among definitions of the marked terms, and the student is advised to read these objects.

Case (c): The system analyses the content of the selected part, and the student is offered a learning object with a similar content.

Case (d): In this case, the system will recommend the student to learn the previous topics.

| Assume that the learner did not understand the concept "second normal form", and he/she chose the option (b). In this case the system, moving on the course ontology, determines that the concepts "functional dependency" and "first normal form" are the parents of the "second normal form". Therefore, the learning materials that describe the parent concepts are recommended for learning (Figure 3).

Below we describe the procedures for the implementation of the above-described actions.

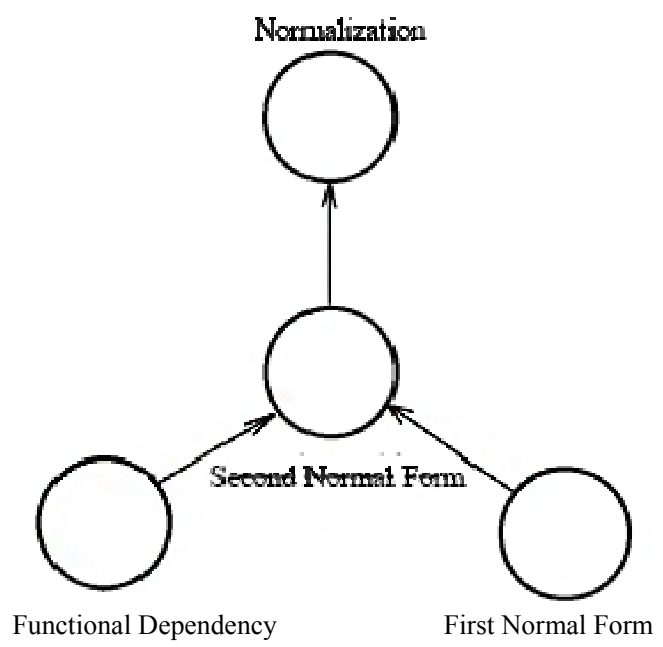

Figure 3. An example of Hierarchic relations between the DBMS concepts

Procedure Understanding Degree (student, course, understanding degree)

input: Student, Course

output: understanding degree

Symbol Definition:

Student

Course

Knowledge level

Topic

Difficulty level

Understanding degree

Predconcepts

Keyconcept

DataBase Tables:

\footnotetext{
$/ *$ the identification number of the student

$/ *$ the identification number of the course, to be learned

$/ *$ the knowledge level of the student

$/ *$ the identification number of the topic, to be learned

$/ *$ the difficulty level of the topic

$/ *$ the understanding degree of the student

$/ *$ the set of predecessor concepts of the concept

${ }^{*}$ key concept of the topic
} 
Student_knowledge_table

Understanding_degree_table

Course_table:
/* contains students profile knowledge

/* contains understanding degrees

/* contains course knowledge

\section{Procedures:}

IRT $\quad{ }^{*}$ calculates the initial value of the understanding degree, depending on learners' knowledge level as well as the difficulty level of the topic to be learned

LTP $\quad / *$ recalculates the understanding degree of the topic by means of the Law of Total Probability, in accordance with the initial understanding degrees of the parent topics, and closeness between the topics and their parents

\section{Actions:}

Retrieve knowledge_level from the learner_profile_table for the student

Convert linguistic value of the knowledge level to the numeric value by using the Table 1

Retrieve difficulty_level from the course_topic_table for the topic

Convert linguistic value of the difficulty level to the numeric value by using the Table 2

Call IRT (knowledge_level, difficulty_level, understanding_degree)

INSERT understanding_degree of the topic for the student INTO learner_course_table

Retrieve keyconcept of the topic from the topic_concept_table

Retrieve understanding_degree from the concept_table for the keyconcept

$u \_$degree_concept $=$understanding degree

Retrieve predconcepts of the concept from the concept ontology

Retrieve understanding_degree for the all predconcepts of the keyconcept from the concept_table

$u \_$degree_predconcept $=$understanding degree

Retrieve the value of closeness between the concept and the predconcepts from the concept ontology

Do I=1 To_ $\mathrm{N}_{\text {predconcept }}$

\{

Call LTP $\left(u \_\right.$degree_predconcept [I], closeness[I], u_degree[I])

$u \_$degree_new $=u \_$degree_new $+u \_$degree [I]

\}

INSERT $u$ _degree-new INTO the learner_course_table

\section{End of Procedure}

Procedure IRT $(\mathrm{T}, \mathrm{B}, \mathrm{P}) / *$ calculate the understanding degree

\section{Symbol Definition}

$\mathrm{T} / *$ ability level; the parameter receives a value from the set of $\{-2,-1,0,1,2\}$

B $/ *$ item difficulty; the parameter receives a value from the set of $\{-2,-1,0,1,2\}$

D $/ *$ scaling constant $(D=1.702)$

\section{Calculate}

$P=E X P(D *(T-B)) /(1+E X P(T-B)) / * P$ means the probability that students with ability $T$ can understand the item with difficulty level $B$

\section{End of the Procedure}

Procedure LTP(PA,PB,PAB) /calculates the understanding degree of the student on the given concept through the Law of Total Probability.

\section{Symbol Definition}

PA $\quad / *$ the probability of the event A

$\mathrm{PB} \quad /^{*}$ the probability of the event $\mathrm{B}$ 
PAB $\quad / *$ the probability of the event A given B

\section{Calculate}

$\mathrm{PA}=\mathrm{PAB} * \mathrm{~PB}$

\section{End of the procedure}

\section{The Experimental Results}

Performance evaluation of the proposed method was carried out on the course of the Database Management System, taught in the Computer Education Department. The following procedure was performed for this purpose.

-First, a detailed list of course topics has been created.

-Then, the keyword concepts and their predecessor concepts have been determined for each topic (Table 3).

-The Concepts Ontology, which includes relationships among about 40 database concepts, has been created.

-The learning materials of the DBMS were selected and saved in the Learning Object Repository.

Grade Point Averages (GPA) of the students were used for estimating the students' knowledge levels.

Table 3. Key concepts and theirs predecessors

\begin{tabular}{|c|c|c|c|c|c|}
\hline \multirow{2}{*}{$\begin{array}{l}\text { Concepts } \\
\text { Normalization }\end{array}$} & \multicolumn{5}{|c|}{ Predecessor Concepts } \\
\hline & $1 \mathrm{NF}$ & $2 \mathrm{NF}$ & $3 \mathrm{NF}$ & relation & attribute \\
\hline $1 \mathrm{NF}$ & UNF & relation & Repeated group & attribute & Key attribute \\
\hline $2 \mathrm{NF}$ & $1 \mathrm{NF}$ & $\begin{array}{l}\text { Functional } \\
\text { dependency }\end{array}$ & Complex key & $\begin{array}{l}\text { Partial functional } \\
\text { dependency }\end{array}$ & $\begin{array}{l}\text { Full functional } \\
\text { dependency }\end{array}$ \\
\hline $3 \mathrm{NF}$ & $1 \mathrm{NF}$ & $2 \mathrm{NF}$ & $\begin{array}{l}\text { Functional } \\
\text { dependency }\end{array}$ & $\begin{array}{l}\text { Transitive functional } \\
\text { dependency }\end{array}$ & Key attribute \\
\hline Data Model & Model & Data & relationship & Data expression & abstraction \\
\hline $\begin{array}{l}\text { Relational } \\
\text { Model }\end{array}$ & Data Model & Relation & attribute & key & Set operations \\
\hline ER diagram & entity & relationship & ER notation & attributes & $\begin{array}{l}\text { Conceptual } \\
\text { model }\end{array}$ \\
\hline EER diagram & ER diagram & EER notation & generalization & specialization & inheritance \\
\hline $\begin{array}{l}\text { Simple SQL } \\
\text { statement }\end{array}$ & Table & SELECT & FROM & WHERE & SQL structure \\
\hline $\begin{array}{l}\text { Relational } \\
\text { Algebra }\end{array}$ & relation & RA operations & RA notation & attributes & tuple \\
\hline $\begin{array}{l}\text { RA Basic } \\
\text { operations }\end{array}$ & Projection & Selection & $\begin{array}{l}\text { Cartesian } \\
\text { Product }\end{array}$ & Intersection & Union \\
\hline
\end{tabular}

The test data consisted of more than 200 students' grades from exams, midterm exams', and semester projects. All of the students were studying at the Computer and Instructional Technology Department, as the study was conducted. On the basis of these data, and observations of the authors, difficulty levels were determined for all of the course topics (Table 4). 
Table 4. Difficulty levels and students average grade points on the topics

\begin{tabular}{lccccc}
\hline Course topics & Difficulty & \multicolumn{4}{c}{ Knowledge level } \\
\hline & Level & low & medium & high & Very high \\
\hline Data Models & $\mathrm{E}$ & 0.32 & 0.82 & 1 & 0.90 \\
Entity-relationship model & $\mathrm{M}$ & 0.41 & 0.63 & 0.81 & 0.92 \\
Extended Entity-relationship model & $\mathrm{D}$ & 0.0 & 0.43 & 0.71 & 0.80 \\
Relational Algebra & $\mathrm{D}$ & 0.0 & 0.41 & 0.70 & 0.91 \\
Relational Model & $\mathrm{M}$ & 0.08 & 0.84 & 0.85 & 0.88 \\
1NF & $\mathrm{M}$ & 0.14 & 0.62 & 0.92 & 1 \\
2NF & $\mathrm{VD}$ & 0.0 & 0.21 & 0.71 & 0.71 \\
3NF & $\mathrm{VD}$ & 0.0 & 0.31 & 0.53 & 0.73 \\
SQL (simple queries) & $\mathrm{E}$ & 0.34 & 0.82 & 0.93 & 1 \\
SQL (complex queries) & $\mathrm{D}$ & 0.22 & 0.50 & 0.80 & 0.90 \\
Query creating in Access DBMS & $\mathrm{VE}$ & 0.45 & 0.94 & 1 & 1 \\
\hline
\end{tabular}

The participants' GPAs and understanding degrees as obtained by means of the proposed APCL method are given in Table 5. Corresponding graphic representations are given in Figure 5. As can be seen in these table and graphics, the exam results, and the results obtained through the APCL method are very close to each other. This indicates the validity of the proposed method.

Table 5. Comparison average grade points of students and the their understanding degrees obtained by the APCL method

\begin{tabular}{lccccccccccc}
\hline \multicolumn{10}{c}{ Difficulty levels of the topics } \\
\hline & \multicolumn{10}{c}{ Very Easy } & \multicolumn{2}{c}{ Easy } & \multicolumn{2}{c}{ Medium } & \multicolumn{2}{c}{ Difficult } & \multicolumn{2}{c}{ Very Difficult } \\
\hline $\begin{array}{l}\text { Students' } \\
\text { knowledge } \\
\text { levels }\end{array}$ & GPA & $\begin{array}{l}\text { Underst. } \\
\text { degree }\end{array}$ & GPA & $\begin{array}{l}\text { Underst. } \\
\text { degree }\end{array}$ & GPA & $\begin{array}{l}\text { Underst. } \\
\text { degree }\end{array}$ & GPA & $\begin{array}{l}\text { Underst. } \\
\text { degree }\end{array}$ & GPA & $\begin{array}{l}\text { Unders. } \\
\text { degree }\end{array}$ \\
\hline VL & 0.05 & 0.50 & 0.01 & 0.27 & 0.00 & 0.12 & 0.00 & 0.05 & 0.00 & 0.01 \\
L & 0.45 & 0.73 & 0.33 & 0.50 & 0.11 & 0.27 & 0.00 & 0.12 & 0.00 & 0.05 \\
M & 0.94 & 0.88 & 0.82 & 0.73 & 0.69 & 0.50 & 0.44 & 0.27 & 0.25 & 0.12 \\
H & 0.99 & 0.95 & 0.93 & 0.88 & 0.86 & 0.73 & 0.73 & 0.50 & 0.62 & 0.27 \\
VH & 0.99 & 0.98 & 0.99 & 0.95 & 0.90 & 0.88 & 0.86 & 0.73 & 0.71 & 0.50 \\
\hline
\end{tabular}




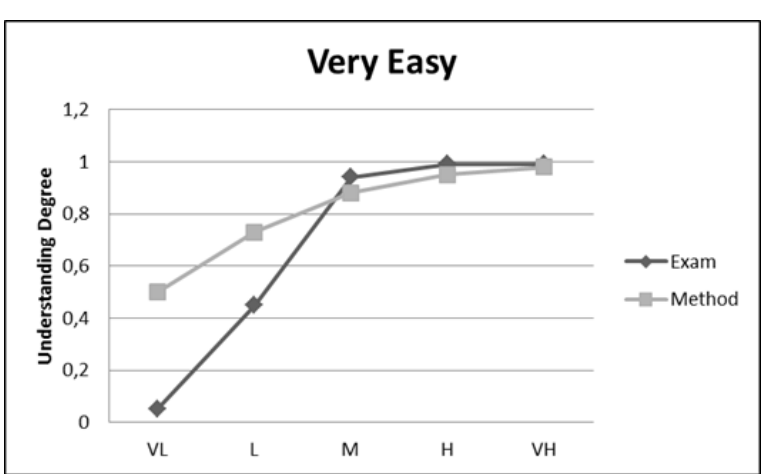

a) difficulty level is "very easy"

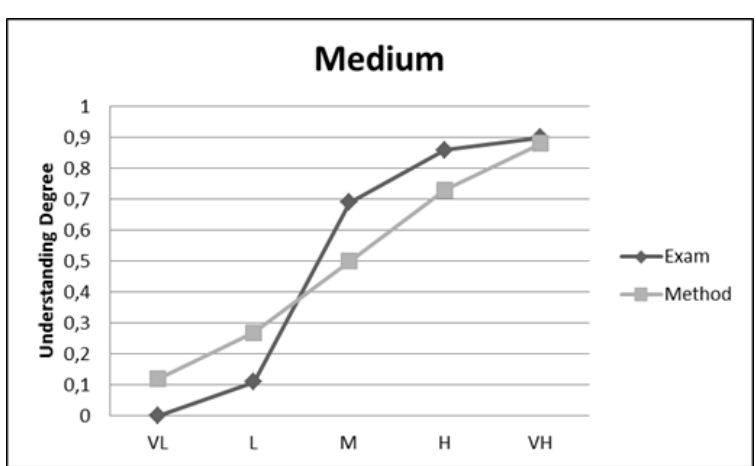

c) difficulty level is "medium"

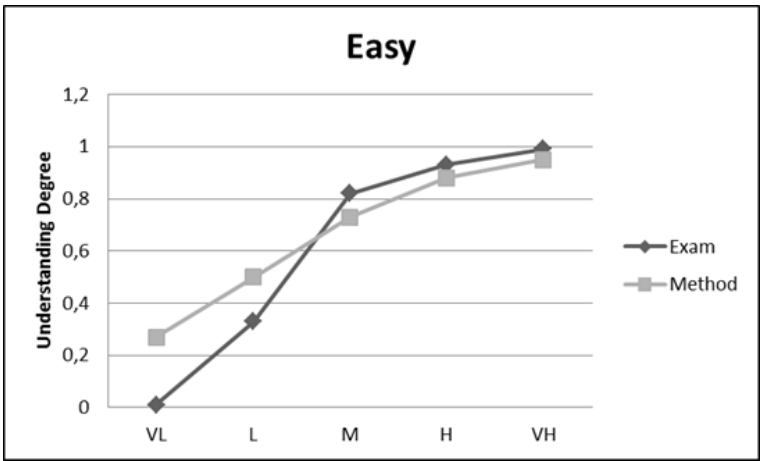

b) difficulty level is "easy"

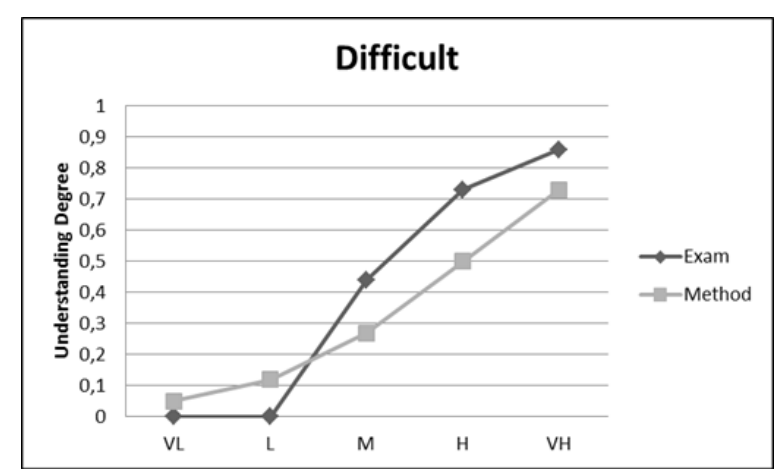

d) difficulty level is "difficult"

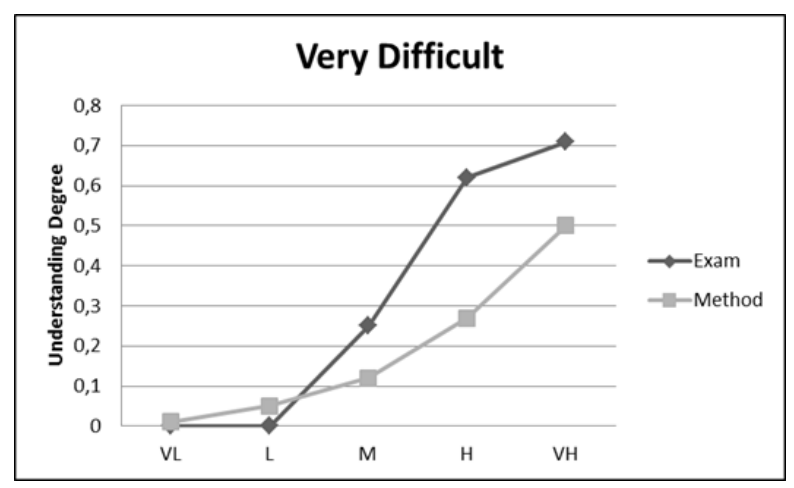

e) Difficulty level is "very difficult"

Figure 5. Comparison of average grades and understanding degrees

The proposed approach has been realised in the Adaptive Personalized Leaning Management System,. The System is realised through Moodle, which is a free software platform for developing Learning Management Systems. Moodle is a software package for producing Internet-based courses and web sites (Moodle, 2012). To realize the proposed approach, functionality of this program has been extended by adding new modules. One screenshot of the APCL application at run-time is given in Figure 6. 


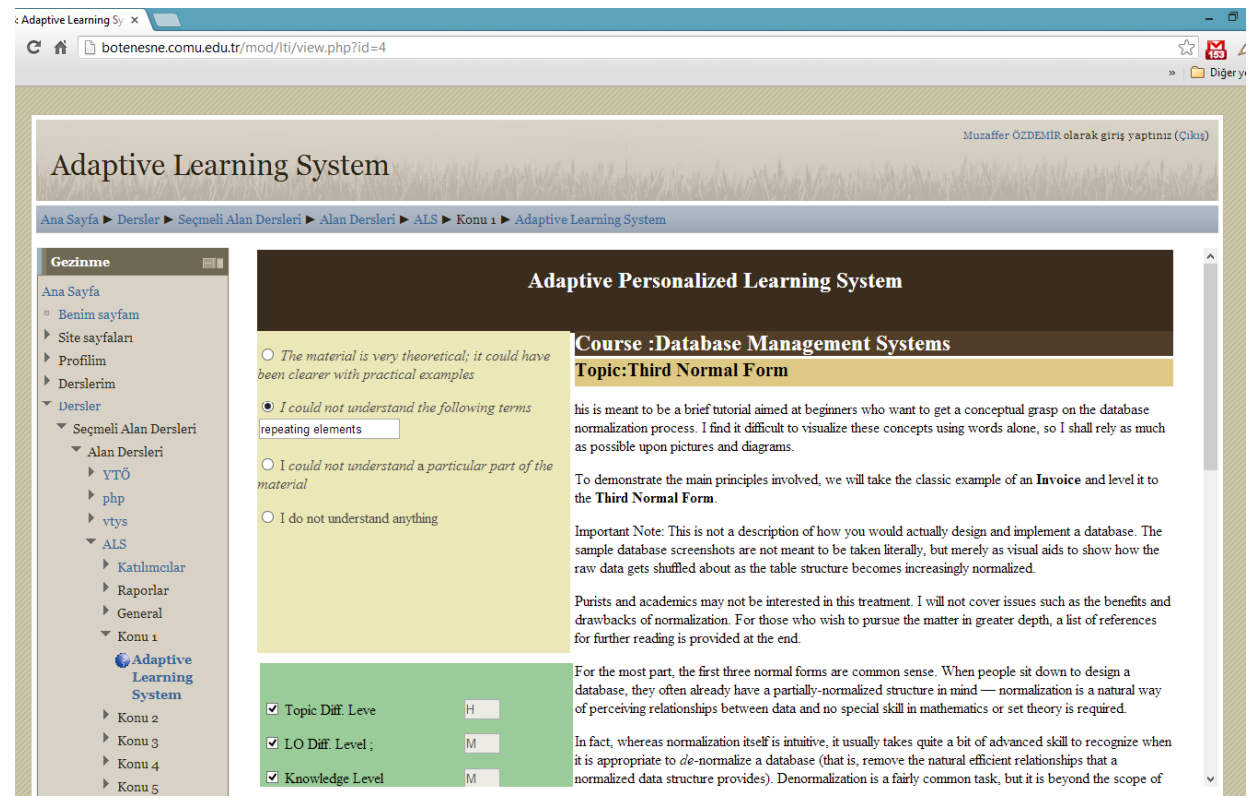

Figure 6. One Sceenshot of the APCL

\section{Conclusion}

In this study an approach was proposed for adaptive personalized course learning system - APCL. The approach is based on the probabilistic models such as the Item Response Theory and The Law of Total Probability, and offers a way of improving learning by increasing the understanding degree of topics to be learned. The main feature of this approach is that adaptively of the learning system is achieved by navigating through not only learning materials, but also the course concepts. For this purpose, a new concept-understanding degree of course topics has been introduced, the value of which depends on the student's knowledge, difficulty of the concepts referred in the topic, as well as the difficulty of the appropriate learning material. The implementation process of the APCL has been described. Furthermore, a description of the system through which the proposed approach has been realised is given. The System has been developed on the Learning Management System Moodle. The program modules that perform adaption functions have been written and added to the Moodle. Performance evaluation of the proposed method was carried out during the course of Database Management System taught by authors in the Computer and Instructional Technology Department. A positive correlation of the relationship between understanding degrees and knowledge levels, and a negative correlation of the relationship between understanding degrees and difficulty levels were confirmed by both methods. Therefore, it would not be wrong to claim that the proposed approach can be successfully applied in the development of adaptive learning systems.

\section{References}

Baker, F. B. (2001). The Basics of Item Response Theory (2nd ed.). Retrieved December 4, 2012, from http://www.eric.ed.gov/PDFS/ED458219.pdf

Baylari, A., \& Montazer, Gh. A. (2009). Design a personalized e-learning system based on item response theory and artificial neural network approach, Expert Systems with Applications, 36(4), 8013-8021. http://dx.doi.org/10.1016/j.eswa.2008.10.080

Chen, C. M., \& Duh, C. M. (2008). Personalized web-based tutoring system based on fuzzy item response theory. Expert Systems with Applications, 34(4), 2298-2315. http://dx.doi.org/10.1016/j.eswa.2007.03.010

Dağ, F., \& Erkan, K. (2010). Personalized Learning System Based on Semantic Web Technologies. Intelligent Tutoring Systems in E-Learning Environments: Design, Implementation and Evaluation, pp. 258-284. http://dx.doi.org/10.4018/978-1-61692-008-1.ch013

Esichaikul, V., Lamnoi, S., \& Bechter, C. (2011). Student Modelling in Adaptive E-Learning Systems. Knowledge Management \& E-Learning: An International Journal, 3(3), 342-355.

Gruber, T. R. (1993). A translation approach to portable ontologies. Knowledge Acquisition, 5(2), 199-220. http://dx.doi.org/http://dx.doi.org/10.1006/knac.1993.1008 
IEEE 1484.12.1-2002 (2002). Draft Standard for Learning Object Metadata, IEEE Learning Technology Standards Committee (LTSC). Retrieved April 9, 2010, from http://ltsc.ieee.org/wg12/files/LOM_1484_12_1_v1_Final_Draft.pdf

Jovanovic, J., Gasevic, D., \& Devedzic, V. (2009). TANGRAM for Personalized Learning Using Semantic Web Technologies. Journal of Emerging Technologies in Web Intelligence, 1(1), 6-21. http://dx.doi.org/10.4304/jetwi.1.1.6-21

Kosba, E., Dimitrova, V., \& Boyle, R. (2003). Fuzzy Student Modeling to Advise Teachers in Web-Based Distance Courses. International Journal of Artificial Intelligence Tools, Special Issue on AI Techniques in Web-Based Educational Systems, World Scientific Net, 13(2), 279-297.

Mödritscher, F., Manuel, V., Barrios, G., \& Gütl., C. (2004). Enhancement of SCORM to support adaptive E-Learning within the Scope of the Research Project AdeLE. In J. Nall, \& R. Robson (Eds.), Proceedings of World Conference on E-Learning in Corporate, Government, Healthcare, and Higher Education. (pp. 2499-2505). Washington, DC, USA, 2004. Retrieved December 4, 2012, from http://www.editlib.org/p/11259

Moodle. (2012). Moodle.org: open-source community-based tools for learning. Retrieved December 4, 2012, from http://moodle.org

Muñoz, L. S., \& Oliveira, J. P. M. (2004). Adaptive web-based courseware development using metadata standards and ontologies. Advanced Information Systems Engineering, Lecture Notes in Computer Science, 3084, 257-279. http://dx.doi.org/10.1007/978-3-540-25975-6_30

Shih, W. C., \& Tseng, S. S. (2009). A Knowledge-based Approach to Retrieving Teaching Materials for Context-aware Learning. Educational Technology \& Society, 12(1), 82-106. Retrieved from http://www.ifets.info/abstract.php?art_id=912

Wikipedia. (2012). Law of total probability-Wikipedia, the free encyclopedia. Retrieved December 4, 2012, from http://en.wikipedia.org/wiki/Law_of_total_probability

Woolf, B. P. (2009). Building intelligent interactive tutors: student-centered strategies for revolutionizing e-learning. Morgan Kaufmann Publishers. ISBN: 9780080920047 